\title{
Recombinant Human BMP-7 Effectively Prevents Non-Union in Both Young and Old Rats
}

\author{
David J. Hak,1 \\ Takeshi Makino,2 \\ Takahiro Niikura, 2 \\ Scott J. Hazelwood,1 \\ Shane Curtiss, 1
}

A. Hari Reddi,1

1Department of Orthopaedic Surgery and Center for Tissue Regeneration and Repair, University of California, Davis, 4860 Y Street, Suite 3800, Sacramento, California 95817

2Department of Orthopaedic Surgery, Kobe University, Kobe, Japan

The purpose of this study was to evaluate the influence of age on the effectiveness of rhBMP-7 treatment in a fracture with severe periosteal damage that is known to result in non-union formation. Closed stabilized femur fractures were produced in 3-month-old and 18-month-old rats. The fracture site was exposed and $2 \mathrm{~mm}$ of the periosteum cauterized circumferentially to impair normal fracture healing. The cauterized fracture site was immediately treated with either $100 \mathrm{mg}$ rhBMP-7 (BMP-7 group), or with 25 $x \mathrm{~L}$ of vehicle alone (control group). Fracture healing was evaluated with radiographs taken at 3 and 6 weeks. Animals were sacrificed at 3 and 6 weeks and specimens subjected to biomechanical and histological evaluation. In both age groups, none of the control animals healed throughout the 6 weeks experimental duration. All of the rhBMP-7-treated 3-month-old animals were radiographically healed at 3 weeks. In comparison, only $56 \%(9 / 16)$ of the rhBMP-7-treated 18 -month-old animals were radiographically healed at 3 weeks. At 6 weeks, however, all of the 18-month-old rhBMP-7-treated animals had healed. Histology revealed slower healing in the 18month-old animals. Treatment with rhBMP-7 significantly increased all of the biomechanical properties in both age groups. In the 3-month-old animals the mechanical strength approached that of the intact femur at 3 weeks, while in the 18-month-old animals this did not occur until 6 weeks. In conclusion, rhBMP-7 can effectively stimulate fracture repair in both young (3- month-old) and old (18-month-old) rats. However, the effect of rhBMP-7 on the rate of fracture healing is greater in young rats 
compared to old rats.

\section{INTRODUCTION}

The rate of fracture healing is progressively slower with increasing age.1 Older patients are at increasing risk for sustaining fractures, with over half of all hospitalizations for fractures occurring in patients age 65 and older. The number of older patients sustaining fractures is projected to rise dramatically as the overall population lifespan increases. It is therefore important to improve our understanding of why fracture healing is delayed in older patients, and develop treatment measures to counteract those detrimental effects.

The ability of bone morphogenetic proteins (BMPs) to enhance fracture healing has been examined in numerous animal and clinical stu-dies.2-6 However, to date there have been limited in vivo studies that have specifically examined the influence of age on the effectiveness of BMP treatment. The purpose of this study was to examine whether age influences the effectiveness of rhBMP-7 treatment in a fracture with severe peristeal disruption that is known to result in nonunion formation.

\section{MATERIALS AND METHODS}

\section{Experimental Design}

Three-month-old (young) and eighteen-month-old (old) Fisher 344 rats were used in this study. The mean body weights were: young, $292 \pm 46$ g; old, $446 \pm 15$ g. All surgical procedures were performed under general anesthesia and normal sterile conditions. Anesthesia was performed with $4 \%$ halothane inhalation, followed by ketamine hydrochloride $(80 \mathrm{mg} / \mathrm{kg})$ and xylazine hydrochloride $(8 \mathrm{mg} / \mathrm{kg})$ administered intraperitoneally.

A lateral parapatellar knee incision on the right limb was made to expose the distal femoral condyle. A 1.25-mm diameter $\mathrm{K}$ wire was inserted from the trochlear groove into the femoral canal in a retrograde fashion with use of a motor-driven drill. The wire was advanced through the greater trochanter and out of the skin until its distal end was positioned deep to the articular surface of the knee. A 5-mm incision in the skin was made around the $\mathrm{K}$ wire and the wire was then cut close to the proximal femur. After irrigation with sterile saline, the wounds were closed with 4-0 nylon suture. A closed transverse femoral shaft fracture was then created in the right femur of each rat using a three-point bending apparatus with a drop weight.7 In order to produce the non-union, the fractured site was then exposed through a lateral approach and the periosteum was cauterized (Loop tip surgical cautery, Abco Dealers Inc., Nashville, TN) circumferentially for a distance of 2 $\mathrm{mm}$ on each side of the fracture. The muscle was protected to preserve all soft tissue except 
the periosteum around the fracture site, and we were careful to cauterize the periosteum only once to prevent excess thermal necrosis of bone. The wound was then irrigated with $10 \mathrm{cc}$ of sterile saline and the wound was dried with gauze.

The rats were randomized to receive one of two treatments. One group was treated with $100 \mathrm{mg}$ rhBMP-7 in $25 \mu \mathrm{L}$ of $50 \mathrm{mM}$ acetic acid containing $3 \mathrm{mg} / \mathrm{mL}$ rat tail tendon collagen (BMP-7 group), and the other group was treated with $25 \mu \mathrm{L}$ of $50 \mathrm{mM}$ acetic acid containing $3 \mathrm{mg} / \mathrm{mL}$ rat tail tendon collagen (control group). The rhBMP-7 or the collagen buffer alone was carefully applied anteriorly, laterally, and posteriorly to the experimental non-union site with a pipet. The viscosity of the collagen buffer prevented extravasation into the surrounding soft tissues. The muscle fascia and skin were closed in layers with 5-0 nylon sutures. Post-operative pain was managed by subcutaneous adminis-tration of buprenorphine hydrochloride. The rats were fed a standard maintenance diet and provided water ad libitum. Unprotected weight bearing was allowed immediately postoperatively.

Eight animals were assigned to each group and were maintained for intervals of 3 and 6 weeks. Five specimens from each group were randomly selected for biomechanical testing at 3 and 6 weeks. The three remaining specimens from each group were processed for histologic examination. Nine animals initially operated on for the study were replaced with animals of like age. Eight animals ( 6 in the old group, 2 in the young group) were excluded because the fracture produced was not a simple transverse fracture, and one young animal died from an anesthetic complication. This research protocol was approved by the Institutional Animal Care and Use Committee.

\section{Radiological Evaluation}

The progress of bone formation was monitored on radiographs taken with a Faxitron imaging device (model 43855A; Faxitron X-ray, Buffalo Grove, IL). Radiographs of all rats were obtained immediately after surgery and at 3 and 6 weeks. This was done under anesthesia with the animal prone and with both limbs fully abducted and externally rotated. Fracture union was determined by the presence of bridging callus on two cortices. Radiographs of each animal were assessed by three blinded independent observers to judge whether the fractures united or not. When the reviewers differed in their interpretation, the classification of healed versus not healed was made by group consensus.

\section{Histological Evaluation}

Three rats from each group were utilized for histological evaluation. After euthanasia, the right femurs were harvested and fixed in $4 \%$ paraformaldehyde in $0.1 \mathrm{M}$ phosphate buffer for $24 \mathrm{~h}$ at $4^{\circ} \mathrm{C}$, defatted in $70 \%$ ethanol, decalcified with $10 \%$ formic acid in citrate for 4 days at $4^{\circ} \mathrm{C}$, and embedded in paraffin. Paraffin sections $4 \mathrm{~mm}$ thick were cut and stained with toluidine blue for histological evaluation. 


\section{Biomechanical Evaluation}

Five animals from each group were used for biomecha-nical evaluation. Following euthanasia the right femurs and the contralateral nonfractured intact femurs were harvested and dissected free of surround-ing muscles. After removal of the intramedullary $\mathrm{K}$ wires, the ends of both femurs were imbedded in Wood's metal (Alfa Aesar, Ward Hill, MA). Specimen lengths were not statistically different between the young control group (19.4 $\pm 3.2 \mathrm{~mm}$ ), the young BMP-7 group $(19.0 \pm 2.9 \mathrm{~mm})$, the old control group (22.0 \pm $2.0 \mathrm{~mm})$, and the old BMP-7 group (21.9 $\pm 1.9 \mathrm{~mm})$. The speci-mens were mounted in a Frankel-Burstein axial torsion-testing machine that was modified to operate under computer control.8 The standard swinging-pendulum mechanism was replaced with an electronic stepper motor drive (model 083062-1-8-031-010, Parker Compumotor, Rohnert Park, CA). Rotational displacement was measured with a precision potentiometer (model 793341-14092, Gould. Inc., Cleveland, OH). Torque was measured with a 100 in. oz. torque cell (model 2105, Eaton Corporation, Troy, MI). Specimens were tested in torsion at a rate of $50^{\circ}$ per minute until failure or through an arc of $45^{\circ}$. Rotational displacement and torque data were collected at $60 \mathrm{~Hz}$ using a digital data acquisition system (model K500A, Keithley Instru-ments, Cleveland, $\mathrm{OH}$ ). Maximum torque to failure was measured directly from the data and torsional stiffness was calculated from a regression of the linear portion of the torque versus angular displacement curve. The energy absorption to maximum torque was also calculated as the area under the curve to the maximum value. The nonfractured contralateral intact femur was also tested to calculate normalized values of maximum torque to failure (percentage maximum torque), stiffness (percentage stiffness), and energy absorption to maximum torque (percentage energy) in order to reduce the influence of individual animal differences.

\section{Statistical Analysis}

A two-factor analysis of variance (ANOVA) was per-formed on the normalized data of the biomechanical properties to account for individual animal differences at each time point ( 3 weeks and 6 weeks). The factors were animal age (young and old group) and treatment (BMP-7 group and control group). A two-factor ANOVA was also performed on the actual values of the biomechanical properties. The factors used were young, old, BMP treated, and intact. Significance was defined as $p$ values less than 0.05 . When appropriate, a Bonferroni-Dunn post hoc test was performed to determine differences between the factors at each time point.

\section{RESULTS}

\section{Radiological Evaluation}


In the 3-month-old animals, all of the rhBMP-7-treated animals were radiographically healed by 3 weeks. In contrast, only 56\% (9/16) of the rhBMP-7-treated 18-month-old animals were radiographically healed at 3 weeks. At 6 weeks, all (8/8) of the older animals achieved radio-graphic union. In both age groups, none of the control animals healed throughout the 6 weeks of experimental duration.

In the young animals the BMP-7 group dis-played abundant callus around the fracture sites at 3 weeks (Fig. 1C). At 6 weeks the callus size had decreased, indicating ongoing remodeling (Fig. 1D). In the older animals, the BMP-7 group also showed callus formation at 3 weeks (Fig. 1G), but it was less robust than in the younger animals (Fig. 1C). At 6 weeks, the size of the callus conti-nued to increase in the older animals (Fig. 1H). No significant callus was seen in either the young or old animals in the control group (Fig. $1 \mathrm{~A}, \mathrm{~B}, \mathrm{E}, \mathrm{F})$. Some callus formed along the periosteum away from the fracture site, but this never extended to bridge the fracture site and a fracture gap still persisted at 6 weeks (Fig. 1B,F).

\section{Histological Evaluation}

At three weeks, the 3-month-old rhBMP-7- treated group displayed an abundant callus formation. Newly formed trabecular bone was the predominant component of this callus. The bony union was less than complete due to the presence of a small amount of cartilage in the callus (Fig. 2C). At 6 weeks, a complete bony union was achieved and the united bone had remodeled as shown by a decrease in the overall diameter of the woven bone (Fig. 2D). The 18-month-old rhBMP-7-treated group displayed bridging cartilaginous tissue at 3 weeks (Fig. 2G). There was a cartilage plate uniting the bony fragments and a few remaining fibrous elements in the fracture gap. Increasing ossification was observed at 6 weeks, however a small amount of cartilaginous elements remained within the cal-lus (Fig. $2 \mathrm{H}$ ).

The control groups of both young and old groups (Fig. 2A,B,E,F) exhibited some nonbridging endo-chondral ossification, however, there was no bone formation at the site of periosteal cauterization. A large gap persisted between the fractured bony fragments even at 6 weeks. Fibrous tissue interposition was observed at the fracture site. Neither bony nor cartilaginous bridging was seen in the control group at either time point.

\section{Biomechanical Evaluation}

The means and standard deviations of the maximum torque to failure, the stiffness, and the energy absorption to maximum torque are shown in Table 1 . Significant increases in all the mechanical properties were seen in the rhBMP-7-treated animals compared to the control ani-mals at each time point in both young and old age groups. When we compared the raw 
values of the rhBMP-7-treated animals with the intact bones at 3 weeks, the values for the old group of rhBMP-7-treated animals were significantly less than the intact bones in maximum torque $(p<0.0001)$ and stiffness $(p<0.0001)$, while there were no significant differences in the young group at 3 weeks. As for energy at 3 weeks, the values of the rhBMP-7-treated animals were significantly less than the intact bones both in the young ( $p=0.0041)$ and old ( $p<0.0001)$ groups. No significant differences were observed at 6 weeks for the young or old groups. The mechanical properties of the control animals remained low in both age groups, indicating development of a nonunion.

To account for variation in animal size, the maximum torque, stiffness, and energy absorption values were also normalized by the respective values of the contralateral intact femurs (percen-tage maximum torque, percentage stiffness, and percentage energy) and average values were calculated for each group (Fig. 3). When we compared the young rhBMP-7 group to the old rhBMP-7 group, we found significant differences in percentage maximum torque ( $p=0.0066$ ) and percentage stiffness ( $p=0.0004)$ at 3 weeks, and no significant differences at 6 weeks.

All the mechanical properties of the rhBMP-7- treated animals increased between 3 and 6 weeks in both age groups with the exception of the percentage stiffness of the young rhBMP-7 group at 3 weeks. The percentage stiffness of the young BMP-7 group already exceeded the stiffness of the intact femur at 3 weeks. At 6 weeks the per-centage maximum torque and percentage energy of the 18-month-old rhBMP-7-treated animals

exceeded $100 \%$, and the percentage stiffness was $97 \%$.

\section{DISCUSSION}

The rate of fracture healing is known to slow with increasing age, but the reason for this deceleration is unknown. The periosteum in child-ren, whose fracture healing is relatively rapid, has a higher cellular and vascular activity. This diminishes with age and older patients display slower cell differentiation.

Few previous in vivo studies have examined fracture healing in aged animals.9-12 In a standard stabilized femur fracture model, younger rats heal more rapidly than older rats. Mechanical strength was restored within 4 weeks in 8-week-old rats, compared with 12 weeks in 32-week-old rats. Meanwhile 50-week-old rats had still not regained their full mechanical strength 24 weeks following fracture.13

Bone induction by both demineralized bone matrix and BMPs has been shown to be decreased in older rats compared to young rats. Reddi reported a significant decline in the osteoinductive potential of bone matrix derived from 24-month-old rats compared to 4month-old rats.14 Fleet and colleagues, using an ectopic bone formation model, reported 
that aging blunts rhBMP-2-induced bone formation in rats.15 They demonstrated a rapid decline in BMP-induced bone formation between the 3-week and 3-month animals, and a further decline between 3 months and 16 months.

Several explanations for the blunted response to BMP in older animals have been proposed. Older mice have been shown to have a smaller number of osteogenic precursor cells and this may limit fracture repair.16 Other studies have shown that the chondrogenic and osteogenic potential of osteoprogenitor cells decrease with increasing age.16,17 Shih and colleagues reported that a reduction in osteoblast recruitment, rather than a reduced capacity to form bone, accounts for the age-related decrease in bone formation.18 Fleet and colleagues proposed that a lower level of circulating or local anabolic agents in older animals may result in fewer available BMP-responsive cells.15 Following treatment with either growth hormone or 1,25-dihydroxyvitamin D3, Fleet and colleagues reported that BMP-induced bone formation in 16-month-old rats returned to that seen in 3-month-old rats. In addition they found that the age-related decline in bone formation could be minimized by increas-ing the amount of rhBMP-2 administered or by extending the length of time the osteoconductive implants were allowed to remain in place. 15 There may also be a deficit of BMP receptors in older animals compared to younger animals. Meyer and colleagues have investigated whether differential expression of various cytokines is a possible explanation for delayed fracture healing seen in aged animals.19-21 They found that osteocalcin, type I and II collagen, BMP-2, BMP-4, and type IA BMP receptor were upregulated following fracture in both young ( 6 week) and old (12-15 month) rats. 21 In the young rats there was elevated gene expression until fracture healing had occurred, while in the older rats the gene expression returned to baseline prior to complete fracture healing. The results of these studies suggest that the age-related impairment in fracture healing is not due to the lack of expression of the various growth factors studied. In addition, the slower healing in older rats did not stimulate a negative-feedback upregulation in gene expression of the various stimulatory cytokines. 19-21

Aronson reported decreased bone formation in older animals in a distraction osteogenesis model in the rat tibia.22 They found a significant reduc-tion in bone formation in the regenerate bone in 24 - month-old rats compared to 9-month-old rats (51\% vs. 68\%). When 24-month-old rats were compared with 3-month-old rats, the reduction was even greater (34\% vs. 95\%). They reported that the periosteal new bone formation was similar in the old and young rats, but the endosteal bone formation was reduced in the older animals.

The possible role for growth factors other than BMP has been examined in aged animal models. Aronson examined the effect of rhFGF administration in old and young rats. Histologically the old rat controls had significantly less endosteal new bone formation than the young rat controls. The old rats treated with rhFGF had significantly increased 
endosteal new bone compared to the old rat controls.9 Blumenfeld and colleagues examined the effect of transforming growth factor 13 (TGF-13) and insulin-like growth factor-1 (IGF-1) in a unicortical bone defect in aged rats. They found that TGF-13 and IGF-1 administered individually improved fracture healing and that the combination of TGF-13 and IGF-1 had a synergistic effect in healing of the bone defect.11

We have developed a reproducible rat femoral non-union model.23 This model was specifically developed to better simulate the altered biological environment that is seen in high energy fractures in which there is severe perisoteal damage leading to non-union formation. Clinical and experimental evidence indicates that periosteal disruption at the fracture site impairs fracture healing.24-26 Even in young 3-month-old rats the severe periosteal damage created in this model impedes normal fracture healing.

We have previously shown that immediate administration of $50 \mu \mathrm{g}$ rhBMP-7 to the fracture site can restore the normal fracture healing process in young 3-month-old rats.27 In the current study we demonstrated that the immediate appli-cation of rhBMP-7 can also restore the healing process in aged animals. Because of the reported blunted effect of BMP response in older animals, we increased the BMP dose from the $50 \mu \mathrm{g}$ we used in younger animals to $100 \mu \mathrm{g}$ in this study. We did not, however, test the lower BMP dose in the older animals in this study. The ability of rhBMP-7 to stimulate bone formation in older animals is important because of the increasing numbers of fractures sustained in older patients.

While not dramatic, the healing rate in the older animals was slowed, even in the presence of rhBMP-7 administration. Serial radiographs indicated that all of the rhBMP-7treated younger animals healed by 3 weeks, while only $56 \%$ of the older animals had united radiographically at 3 weeks. The mechanical results also demonstrated slower restoration in the older animals. In the older animals, the actual maximum torque ( $p<$ 0.0001 ) and stiffness ( $p=0.0001$ ) values of the rhBMP-7-treated group were significantly less than the intact bones at 3 weeks, while there were no significant differences in the younger animals at this time point. At 6 weeks there were no significant differences between the BMP-treated groups and the intact bone in either age group.

In conclusion, the present study demonstrates that the immediate application of rhBMP7 has the potential to accelerate fracture healing in older animals with impaired fracture healing, even in the presence of significant periosteal damage. However, the effect of rhBMP-7 on the rate of fracture healing is greater in young rats compared to old rats.

\section{ACKNOWLEDGMENTS}

This work was supported by a grant from the Orthopaedic Research and Education Foundation. rhBMP-7 was a generous gift from Dr. T. K. Sampath (Creative BioMolecules, Hopkinton, MA). 


\section{REFERENCES}

1. Nilsson BE, Edwards P. 1969. Age and fracture healing: a statistical analysis of 418 cases of tibial shaft fractures. Geriatrics 24:112-117.

2. Cook SD, Wolfe MW, Salkeld SL, et al. 1995. Effect of recombinant human osteogenic protein-1 on healing of segmental defects in non-human pri-mates. J Bone Joint Surg Am 77A:734-750.

3. Friedlaender GE, Perry CR, Cole JD, et al. 2001. Osteogenic protein-1 (bone morphogenetic protein-7) in the treatment of tibial nonunions. J Bone Joint Surg Am 83A(Suppl 1):S151S158.

4. Geesink RG, Hoefnagels NH, Bustra SK. 1999. Osteogenic activity of OP-1 bone morphogenetic protein (BMP-7) in a human fibular defect. J Bone Joint Surg Br 81B:710718.

5. Louwerse RT, Heyligers IC, Klein-Nulend J, et al. 2000. Use of recombinant human osteogenic protein-1 for the repair of subchondral defects in articular cartilage in goats. J Biomed Mater Res 49:506-516.

6. Mizumoto Y, Moseley T, Drews M, et al. 2003. Acceleration of regenerate ossification during dis-traction osteogenesis with recombinant human bone morphogenetic protein-7. J Bone Joint Surg Am 85A:S151-S158.

7. Bonnarens F, Einhorn TA. 1984. Production of a standard closed fracture in laboratory animal bone. J Orthop Res 2:97-101.

8. Burstein AH, Frankel VH. 1971. A standard test for laboratory animal bone. J Biomech 4:155-158.

9. Aronson J. 2004. Modulation of distraction osteo-genesis in the aged rat by fibroblast growth factor. Clin Orthop 425:264-283.

10. Bak B, Andreassen TT. 1991. The effect of growth hormone on fracture healing in old rats. Bone 12:151-154.

11. Blumenfeld I, Srouji S, Lanir Y, et al. 2002. Enhancement of bone defect healing in old rats by TGF-3 and IGF-1. Exp Gerontol 37:553-565.

12. Delgado-Martinez AD, Martinez ME, Carrascal MT, et al. 1998. Effect of 25-OH-Vitamin D on fracture healing in elderly rats. J Orthop Res 16:650-653.

13. Meyer RA Jr, Tsahakis PJ, Martin DF, et al. 2001. Age and ovariectomy impair both the normalization of mechanical properties and the accretion of mineral by the fracture callus in rats. J Orthop Res 19:428-435.

14. Reddi AH. 1985. Age-dependent decline in extra-cellular matrix-induced local bone differentiation. Isr J Med Sci 21:312-313.

15. Fleet JC, Cashman K, Cox K, et al. 1996. The effects of aging on the bone inductive activity of recombinant human bone morphogenetic protein-2. Endocrinology 137:4605-4610.

16. Bergman RJ, Gazit D, Kahn AJ, et al. 1996. Age-related changes in osteogenic stem cells in 
mice. J Bone Mineral Res 11:568-577.

17. O'Driscoll SWM, Saris DBF, Ito Y, et al. 2001. The chondrogenci potential of periosteum decreases with age. J Orthop Res 19:95-103.

18. Shih MS, Cook MA, Spence CA, et al. 1993. Relationship between bone formation rate and osteoblast surface on different subdivisions of the endosteal envelope in aging and osteoporosis. Bone 14:519-521.

19. Desai BJ, Meyer MH, Porter S, et al. 2003. The effect of age on gene expression in adult and juvenile rats following femoral fracture. J Orthop Trauma 17:689-698.

20. Meyer RA Jr, Meyer MH, Tenholder M, et al. 2003. Gene expression in older rats with delayed union of femoral fractures. J Bone Joint Surg 85A:1243- 1254.

21. Meyer RA, Meyer MH, Phieffer LS, et al. 2001. Delayed union of femoral fractures in older rats: decreased gene expression. BMC Musculoskel Disord 2:2.

22. Aronson J, Gao GG, Shen XC, et al. 2001. The effect of aging on distraction osteogenesis in the rat. J Orthop Res 19:421-427.

23. Kokubu T, Hak DJ, Hazelwood SJ, et al. 2003. Development of an atrophic nonunion model and comparison to a closed healing fracture in rat femur. J Orthop Res 21:503-510.

24. McKibbin B. 1978. The biology of fracture healing in long bones. J Bone Joint Surg Br 60B:150- 162.

25.Oni OO, Stafford H, Gregg PJ. 1989. An experi-mental study of the patterns of periosteal and endosteal damage in tibial shaft fractures using a rabbit trauma model. J Orthop Trauma 3:142- 147.

26.Park SH, O'Connor K, Sung R, et al. 1999. Comparison of healing process in open osteotomy model and closed fracture model. J Orthop Trauma 13:114-120.

27. Makino T, Hak DJ, Hazelwood SJ, et al. 2005. Prevention of atrophic nonunion development by recombinant bone morphogenetic protein-7. J Orthop Res (in press). 

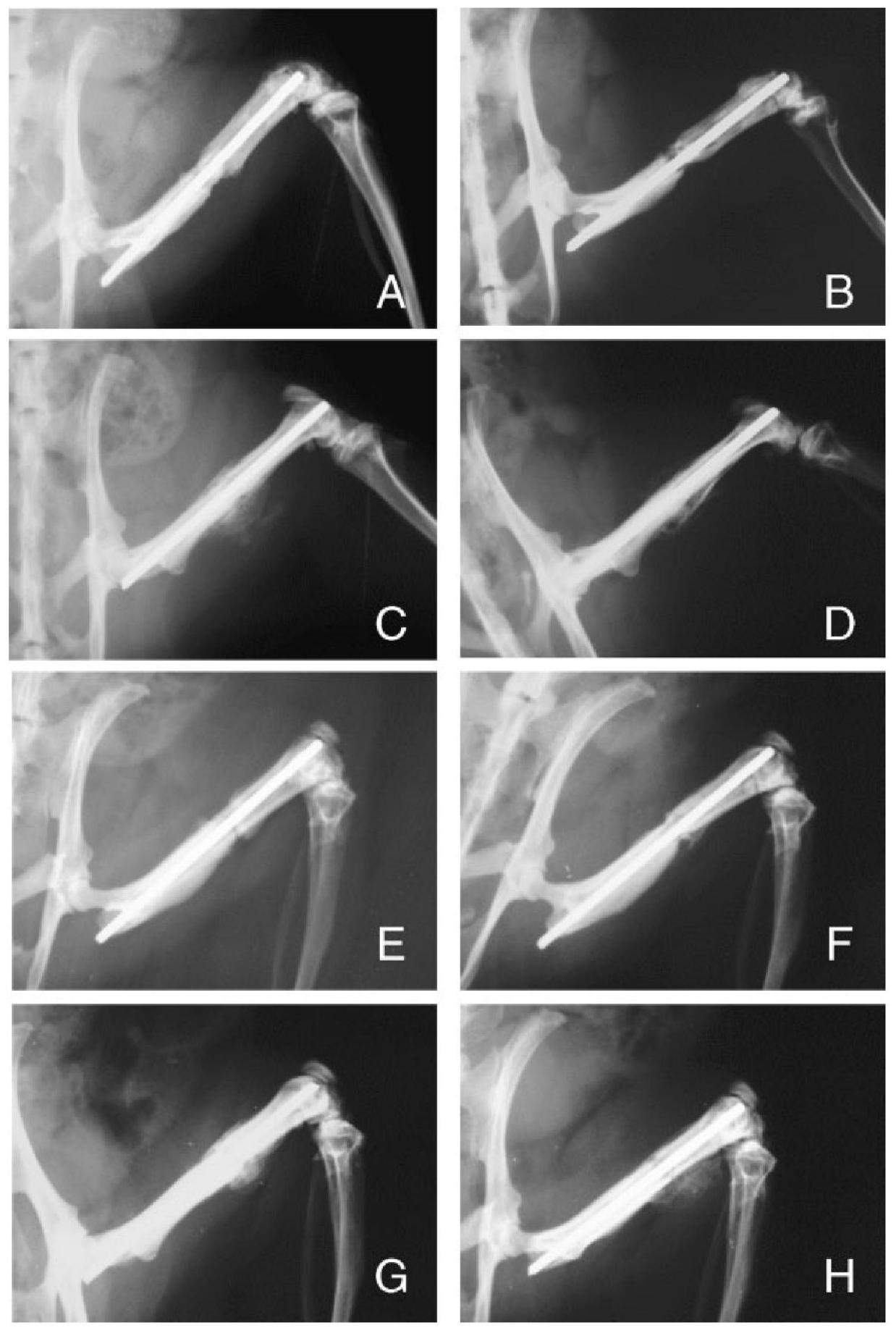

Figure 1. Radiographs of the young control group (A, B), the young rhBMP-7 group (C, D), the old control group (E, F), and the old rhBMP-7 group (G, H) obtained at 3 weeks after surgery (A, C, E, G) and 6 weeks after surgery (B, D, F, H). Note the abundant callus at 3 weeks (C) and the reduced amounts of callus at 6 weeks (D) in the young rhBMP-7 group. On the other hand, more abundant callus is observed at 6 weeks $(\mathrm{H})$ than at 3 weeks (G) in the old rhBMP-7 group. Atrophic non-union developments are seen at 6 weeks in the control group of both young and old (B, F). 

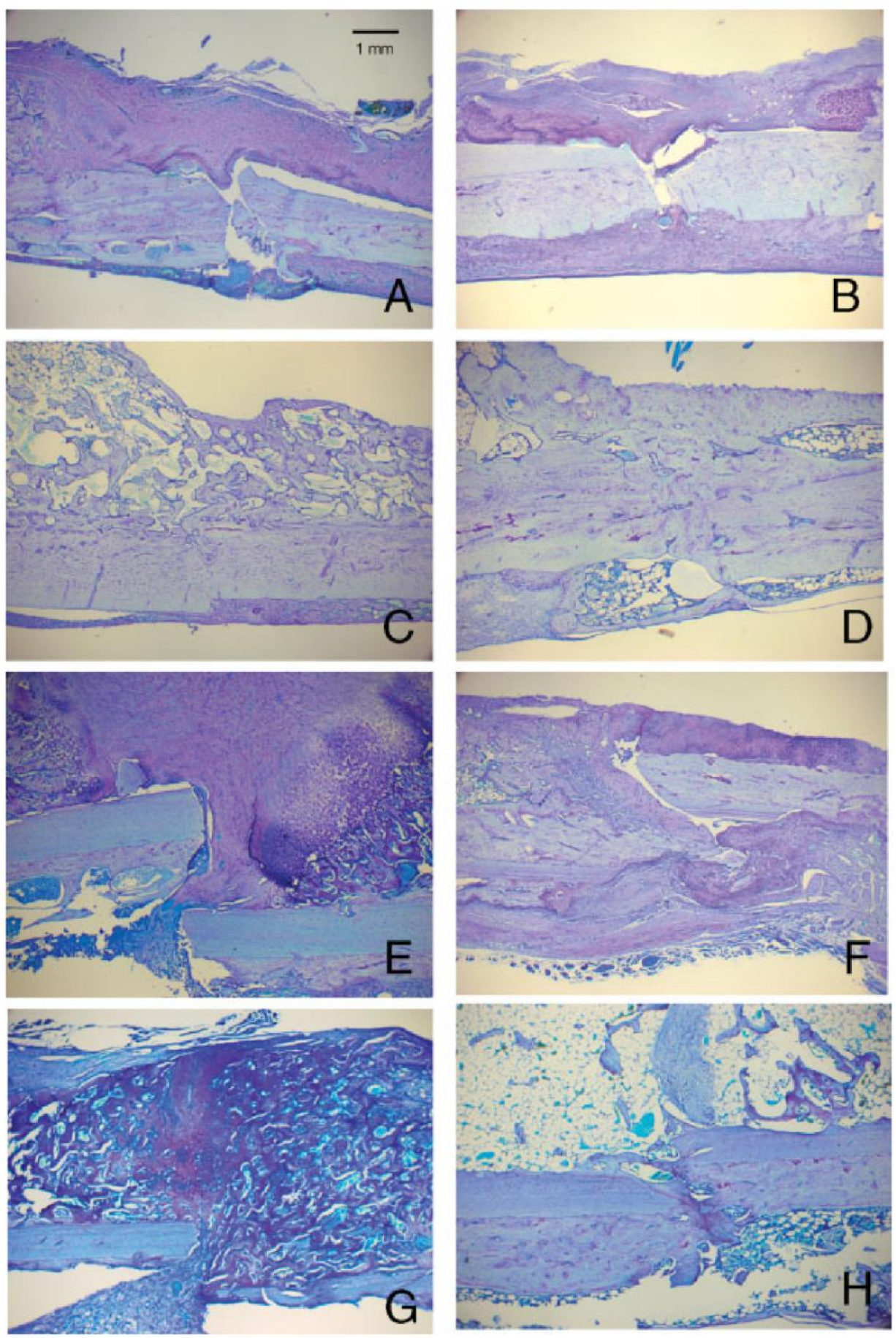

Figure 2. Histological findings of the young control group (A, B), the young rhBMP-7 group (C, D), the old control group (E, F), and the old rhBMP-7 group (G, H). Sections were stained with toluidine blue. The young rhBMP-7 group at 3 weeks displayed an incomplete bony union (C), and a complete bony union at 6 weeks (D). The old rhBMP-7 group displayed a cartilaginous union at 3 weeks $(G)$, and an incomplete bony union at 6 weeks $(H)$. Control groups obtained neither bony nor cartilaginous union even though at 6 weeks. $\mathrm{Bar}=1 \mathrm{~mm}$. 
Table 1. Mechanical Test Results of 3-Month-Old and 18-Month-Old Animals

\begin{tabular}{|c|c|c|c|c|}
\hline & & Intact & Control & BMP-7 \\
\hline \multicolumn{5}{|c|}{ Maximum torque to failure (nm) } \\
\hline \multirow[t]{2}{*}{ 3-month-old animals } & 3 weeks & $0.295 \pm 0.037$ & $0.015 \pm 0.009$ & $0.239 \pm 0.020$ \\
\hline & 6 weeks & $0.421 \pm 0.039$ & $0.028 \pm 0.014$ & $0.418 \pm 0.049$ \\
\hline \multirow[t]{2}{*}{ 18-month-old animals } & 3 weeks & $0.425 \pm 0.142$ & $0.033 \pm 0.030$ & $0.285 \pm 0.073$ \\
\hline & 6 weeks & $0.471 \pm 0.169$ & $0.072 \pm 0.057$ & $0.493 \pm 0.081$ \\
\hline \multicolumn{5}{|l|}{ Stiffness (nm/rad) } \\
\hline \multirow[t]{2}{*}{ 3-month-old animals } & 3 weeks & $1.198 \pm 0.240$ & $0.036 \pm 0.030$ & $1.115 \pm 0.088$ \\
\hline & 6 weeks & $1.747 \pm 0.202$ & $0.070 \pm 0.053$ & $1.826 \pm 0.247$ \\
\hline \multirow[t]{2}{*}{ 18-month-old animals } & 3 weeks & $2.353 \pm 0.341$ & $0.050 \pm 0.031$ & $1.492 \pm 0.348$ \\
\hline & 6 weeks & $2.608 \pm 0.443$ & $0.286 \pm 0.222$ & $2.151 \pm 0.392$ \\
\hline \multicolumn{5}{|c|}{$\begin{array}{l}\text { Energy absorption to maximum } \\
\text { torque (nm rad) }\end{array}$} \\
\hline \multirow[t]{2}{*}{ 3-month-old animals } & 3 weeks & $0.044 \pm 0.005$ & $0.002 \pm 0.002$ & $0.029 \pm 0.004$ \\
\hline & 6 weeks & $0.057 \pm 0.009$ & $0.004 \pm 0.003$ & $0.066 \pm 0.014$ \\
\hline \multirow[t]{2}{*}{ 18-month-old animals } & 3 weeks & $0.049 \pm 0.029$ & $0.006 \pm 0.005$ & $0.028 \pm 0.011$ \\
\hline & 6 weeks & $0.058 \pm 0.043$ & $0.007 \pm 0.006$ & $0.083 \pm 0.037$ \\
\hline
\end{tabular}

Figure 3. Biomechanical properties of the rhBMP-7 group and the control group in both young and old groups normalized by the respective values of the intact femurs. (A) Percentage maximum torque (maximum torque to failure of fractured femur/nonfractured intact femur $\times 100$ ). (B) Percentage stiffness (stiffness of fractured femur/ nonfractured intact femur $\times 100$ ). (C) Percentage energy (energy absorption to maximum torque/nonfractured intact femur $\times 100$ ). Significant increases in all the mechanical properties were seen in the rhBMP-7 group compared to the control at each time points both in young and old groups. Comparing the young rhBMP-7 group and the old rhBMP-7 group, we found significant differences in percentage maximum torque $(p=0.0066)$ and percentage stiffness $(p=0.0004)$ at 3 weeks, and no significant differences at 6 weeks. ${ }^{*} p<0.01,{ }^{* *} p<0.001,{ }^{* * *} p<0.0001$. 
A

Percent Maximum Torque

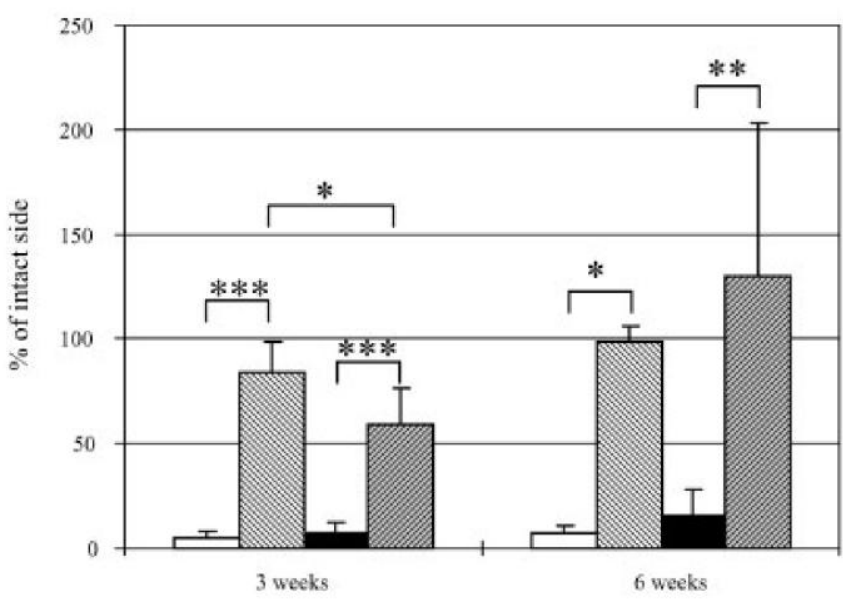

Young Control

⿶ Young th BMP-7

- Old Control

징 Old BMP-7

B

Percent Stiffness

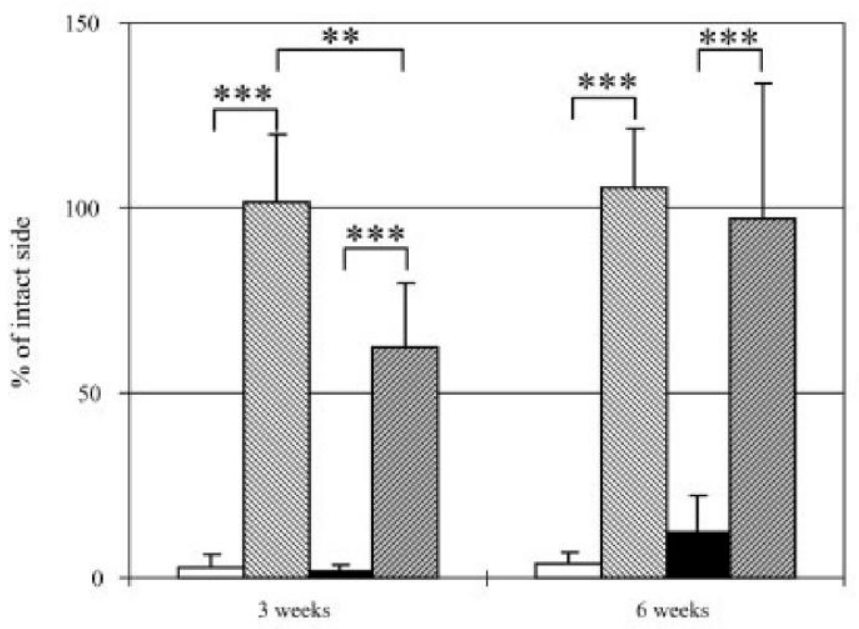

$\square$ Young Control

Young rh BMP-7

- Old Control

ש Old rh BMP-7

C

Percent Energy

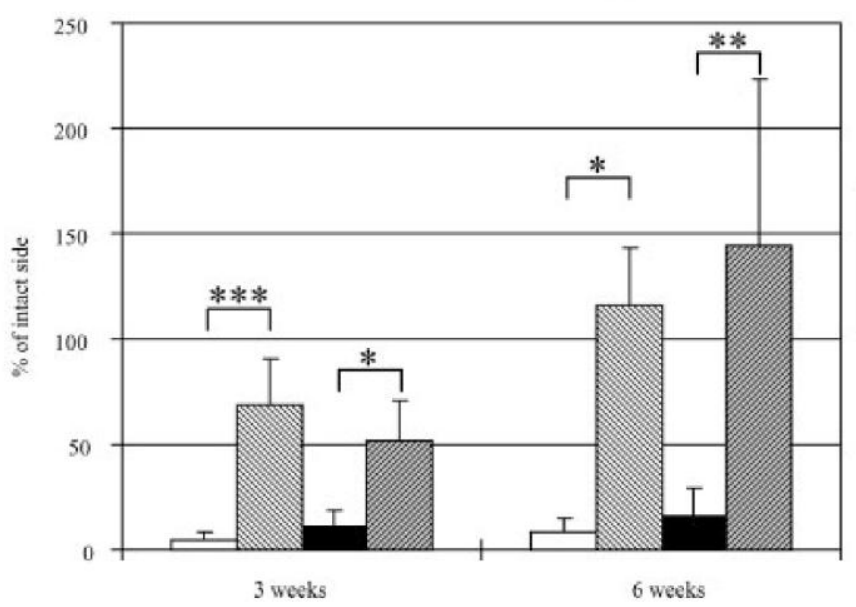

ㅁ Young Control

ه Young ih BMP-7

- Old Control

ש Old rh BMP-7

Figure 3. 